\title{
SHARP ERROR BOUNDS FOR TURNING POINT EXPANSIONS
}

\author{
T. M. Dunster*, A. Gil And J. SEgurA
}

\begin{abstract}
Computable and sharp error bounds are derived for asymptotic expansions for linear differential equations having a simple turning point. The expansions involve Airy functions and slowly varying coefficient functions. The sharpness of the bounds is illustrated numerically with an application to Bessel functions of large order.
\end{abstract}

Mathematics subject classification (2020): 34E05, 33C10, 33F05, 34E20.

Keywords and phrases: Asymptotic expansions, airy functions, turning point theory, WKB methods.

\section{REFERENCES}

[1] F. Bornemann, Accuracy and stability of computing high-order derivatives of analytic functions by Cauchy integrals, Found. Comput. Math., 11 (2011), p. 1-63, https://doi.org/10.1007/s10208-010-9075-z.

[2] NIST Digital Library of Mathematical Functions, http://dlmf .nist.gov/, Release 1.1.1 of 202103-15, http://dlmf .nist.gov/. F. W. J. Olver, A. B. Olde Daalhuis, D. W. Lozier, B. I. Schneider, R. F. Boisvert, C. W. Clark, B. R. Miller, B. V. Saunders, H. S. Cohl, and M. A. McClain, eds.

[3] T. M. DUNSTER, Asymptotic solutions of inhomogeneous differential equations having a turning point, Stud. Appl. Math., 145 (2020), pp. 500-536, https ://doi .org/10.1111/sapm. 12326.

[4] T. M. DUNSTER, Liouville-Green expansions of exponential form, with an application to modified Bessel functions, Proc. Roy. Soc. Edinburgh Sec. A, 150 (2020), pp. 1289-1311 https://doi.org/10.1017/prm.2018.117.

[5] T. M. Dunster, A. Gil, AND J. Segura, Computation of asymptotic expansions of turning point problems via Cauchy's integral formula: Bessel functions, Constr. Approx., 46 (2017), pp. 645-675, https://doi.org/10.1007/s00365-017-9372-8.

[6] T. M. Dunster, A. GiL, And J. Segura, Simplified error bounds for turning point expansions, Anal. Appl., 19 (2021), pp. 647-678, https ://doi .org/10.1142/S0219530520500104.

[7] F. W. J. Olver, Asymptotic approximations and error bounds, SIAM Review, 22 (1980), pp. 188203, https://doi.org/10.1137/1022028.

[8] F. W. J. Olver, Asymptotics and special functions, AKP Classics, A K Peters Ltd., Wellesley, MA, 1997. Reprint of the 1974 original [Academic Press, New York].

[9] B. SURY, T. WANG, AND F.-Z. ZHAO, Identities involving reciprocals of binomial coefficients, J. Integer Seq., 7 (2004), http://eudml.org/doc/51545. 